\title{
Electron Density Enhancement During an UV Explosive Event
}

\author{
M. S. Madjarska, J. G. Doyle and L. Teriaca \\ Armagh Observatory, Armagh BT61 9DG, N. Ireland
}

\begin{abstract}
High resolution temporal observations performed with the SUMER spectrometer on SoHO provide us the opportunity to investigate the electron density variations in the solar mid-transition region due to explosive event-like phenomena. The O IV 1401.16/1404.81 density sensitive line intensity ratio shows a clear increase during a strong explosive event, corresponding to an electron density enhancement of a factor of $\sim 3$ with respect to pre-event values. This is consistent with recent MHD simulations (2.5 D).
\end{abstract}

\section{Introduction}

The explosive events were described for the first time by Brueckner \& Bartoe (1983) as high energy events seen in lines from ions formed at transition region temperatures (particularly C IV and Si IV). They are preferentially observed in the network lanes (Porter \& Dere 1991), in regions with weak and mixed polarity fluxes displaying magnetic neutral lines (Chae et al. 1998). High velocity components with typical values of $100 \mathrm{~km} \mathrm{~s}^{-1}$, average lifetimes of $\sim 60 \mathrm{~s}$ and spatial dimensions of $\sim 2000 \mathrm{~km}$ are typically observed (Innes et al. 1997a). Dere (1994), using the ratio of O IV lines, reported an electron density of $710^{10} \mathrm{~cm}^{-3}$ in an explosive event. More recent indications of density enhancement during explosive events has been presented by Wilhelm et al. (1998). In the present contribution we studied the behaviour of the O IV 1401.16/1404.81 line ratio during the appearance of an UV explosive event.

\section{Observational material}

The dataset analysed in this work was obtained on the 'quiet Sun' in a sit-andstare mode with no compensation of solar rotation applied. It consists of 42 spectra centered at $X=3$ and $Y=0$, obtained exposing for 20 seconds the central part of detector A through the $1 \times 120 \operatorname{arcsec}^{2}$ slit. For each spectrum, four spectral windows (50 pixels wide) were transmitted to the ground, respectively centered on O IV $1399.77 \AA$, O IV $1401.16 \AA$, O IV $1404.81 \AA$ and O IV $1407.38 \AA$. The data were reduced using the standard SUMER analysis software.

\section{Data analysis}

The explosive event was identified through the presence of the left wing of the strong Si IV $1402 \AA$ line into the spectral window centered on the O IV 1401.16 
$\AA$ line (see Fig. 1h). A closer analysis reveals that the event is visible also in the O IV 1401.16 line. A region 7 pixels wide was hence selected along the slit and, binning over different time intervals, the parameters of the lines of interest were determined.

\section{Electron density determination}

To derive the electron density, the most reliable method involves the use of density-sensitive intensity ratio of lines from the same ion (Wikstøl et al. 1998). In this study the $\mathrm{O}$ IV $1401.16 / \mathrm{O}$ IV 1404.81 line ratio is used. The major difficulties with these lines are due to the presence of line blends. The blend between the O IV $1401.16 \AA$ and the S I $1401.51 \AA$ lines is easily resolved through a double Gaussian fit. In the case of the O IV $1404.81 \AA$ line, the use of an atomic database is required in order to obtain the contribution of S IV $1404.771 \AA$ and $\mathrm{O}$ III $702.337 \AA$ lines. Using the CHIANTI database (Landi et al. 1999) the theoretical ratios O III 702.337/703.85 and S IV 1404.77/1406.08 were calculated, finding values of 0.265 and 0.21 , respectively. The intensities of the $\mathrm{O}$ III 703.85 and S IV 1406.08 were obtained through multi-Gaussian fits. It was found that the average contributions of O III $702.34 \AA$ and S IV $1404.77 \AA$ to the total 1404 spectral feature are $39 \%$ and $4 \%$, respectively. The intensity line ratio of $\mathrm{O}$ IV $1401.16 \AA$ to $\mathrm{O}$ IV $1404.81 \AA$ was translated to electron density using the CHIANTI database at the $\mathrm{O}$ IV formation temperature $\log T_{e}=5.2$.

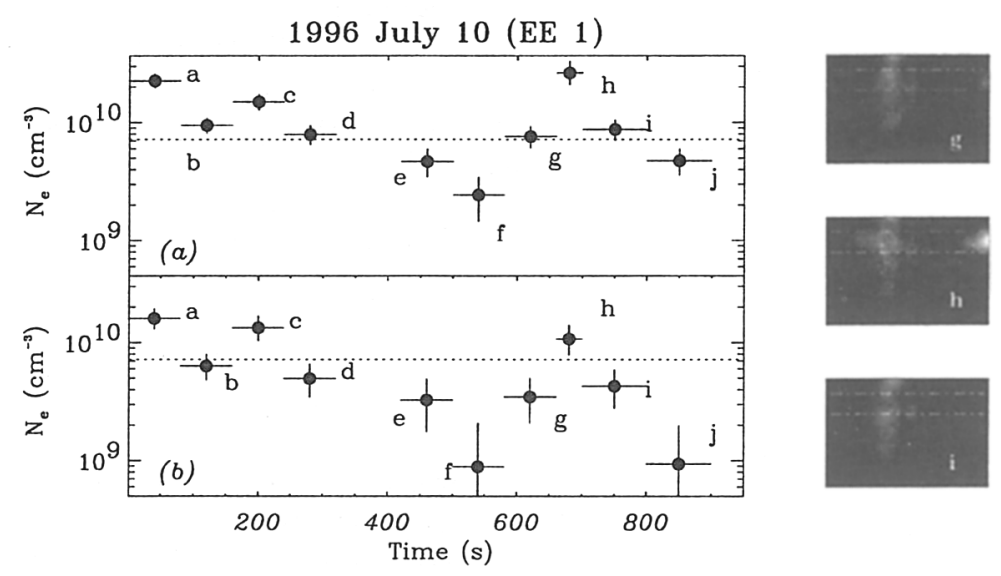

Figure 1. The horizontal dashed lines indicate the average electron density over the whole dataset. Vertical bars indicate errors in density, while the horizontal ones indicate the integrated time interval. (a): Values obtained assuming a fixed $57 \%$ contribution of O IV $1404.81 \AA$ on the whole $1404 \AA$ feature. (b): Values obtained estimating in each point the contribution of O IV $1404.81 \AA$ on the whole $1404 \AA$ feature through the O III $703.85 \AA$ line. Left panels: Slit images before $(g)$, during (h) and after (i) the explosive event. 


\section{Results and discussion}

An average over the whole dataset was performed obtaining an electron density of $\left(7.2_{-0.6}^{+0.7}\right) \times 10^{9} \mathrm{~cm}^{-3}$ that can be considered as typical for the quiet Sun. The density in the selected region was calculated assuming a constant $57 \%$ contribution of O IV 1404.81 for all points (Fig. 1a) and estimating at each point the importance of the line blends (Fig. 1b). In both cases, an electron density enhancement of a factor of $\sim 3$ is evident in Fig. 1 at point $h$ with respect to pre-event values (Fig. 1, $g$ and $i$ ). The presence of bi-directional jets (Innes et al. 1997b) with velocities comparable to the local Alfvén speed, together with the often observed association with episodes of photospheric magnetic flux cancellation (Chae et al. 1998) suggests a sequence of magnetic reconnection events (Parker 1988; Porter \& Dere 1991; Dere 1994; Innes et al. 1997b). Recent MHD modelling of shear-induced magnetic reconnection between two magnetic dipoles, carried out by Karpen et al. (1998), shows the appearance of features in which the density is 4-10 times higher than the pre-event local electron density. Also, Roussev et al. (2000) have recently presented results on the modelling of reconnecting jets again finding electron density increases of a factor of 2-4 in the transition region.

\section{Conclusions}

We find evidence of an electron density increase associated with a UV explosive event, consistent with recent MHD simulations of magnetic reconnection. Our results thus constitute further support for magnetic reconnection as the cause of UV explosive events.

Acknowledgments. Research at Armagh Observatory is grant-aided by the N. Ireland Dept. of Culture, Arts and Leisure, while partial support for software and hardware is provided by the STARLINK Project which is funded by the UK PPARC. This work was supported by PPARC grant PPA/G/S/1999/00055.

\section{References}

Chae J., Wang H., Lee C.Y., Goode P.R., Schühle U., 1998, ApJ 497, L109 Dere K.P., Bartoe J.-D.F., Brueckner G.E., 1989, Solar Phys. 123, 41

Dere K.P., 1994, Adv. Space Res. 14(4), 13

Innes D.E., Brekke P., Germerott D., Wilhelm K., 1997a, Solar Phys. 175, 341

Innes D.E., Inhester B., Axford W.I., Wilhelm K., 1997b, Nature 386, 811

Karpen J.T., Antiochos S.K., DeVore C.R., Golub L., 1998, ApJ 495, 491

Landi E., Landini M., Dere K.P.et al., 1999, A\&AS 135, 339

Porter J.G., Dere K. P., 1991, ApJ 370, 775

Roussev I., Galsgaard K., Erdélyi R., Doyle J.G., 2000, A\&A (submitted)

Wikstøl Ø., Judge P. G., Hansteen V., 1998, ApJ 501, 895

Wilhelm K., Innes D.E., Curdt W., Kliem B., Brekke P., 1998, ESA-SP 421, 103 